\title{
Zona de Desarrollo Próximo como eje del desarrollo de los estu- diantes: de la ayuda a la colaboración
}

\section{Proximal zone of development as axis of development of students: from sup- port to collaboration}

\author{
Alberto Félix Labarrere Sarduy* \\ Universidad Santo Tomás, Santiago, Chile \\ (Rec.: mayo de 2016 - Acept.: junio de 2016)
}

\begin{abstract}
Resumen
El presente material constituye una aproximación al legado y pensamiento de S. L. Vigotsky. En él se aborda la noción de Zona de Desarrollo Próximo (ZDP), se discute su lugar en el pensamiento de Vigotsky y se avala su trascendencia para la enseñanza y el aprendizaje. Se intenta una aproximación a la acción didáctica formativa basada en la categoría desarrollo como eje de la preocupación vigotskiana. Adicionalmente, se enuncia la noción de Situación de Enseñanza y Aprendizaje Orientada al Desarrollo (SEAOD), de la cual se formulan sus componentes funcionales y estructurales. Se ofrecen ejemplos a manera de ilustrar el trabajo en SEAOD.
\end{abstract}

Palabras clave: Zona de desarrollo próximo; enseñanza, aprendizaje y desarrollo, situación enseñanza aprendizaje orientada al desarrollo.

\begin{abstract}
The present work contains an approximation to Vygotsky's legacy and thought. Notion of Proximal Zone of Development (PZD) is presented. Its place in Vygotsky's thought as well as its significance for teaching and learning are not only discussed but also endorsed. An approach to didactic action based on the category of development (as a central concept in vigostkian thought) is attempted. Additionally, a notion of Teaching and Learning Development-Oriented Situation (TLDOS) is enunciated and its functional and structural components are formulated. Finally, some examples are provided to illustrate the work in TLDOS.
\end{abstract}

Keywords: Proximal zone of development, teaching, learning, teaching and learning development-oriented situation.

\footnotetext{
* Correspondencia a: Alberto Félix Labarrere Sarduy. Escuela de Psicología. Facultad de Ciencias Sociales. Universidad Santo Tomás, sede Ejército. Ejército 146, Santiago de Chile. Teléfonos 56223624892 - 562 3624888. Email: alabarrere@santotomas.cl.
} 


\section{En el contexto del legado de Vigotsky}

Esta fuera de cualquier duda, la brillantez de Vigotsky en su aproximación a la gran multiplicidad de problemas que abordó durante su corta vida y el tiempo que dedicó a la producción científica. Las soluciones, los caminos por él abiertos, las interrogantes que dejó para la Psicología y, de manera general, los desafíos que planteó constituyen un legado muy importante y útil para todo aquel que pretenda adentrase en la comprensión del proceso educativo.

Existen múltiples maneras de aproximarse y apropiarse de dicho legado. Aunque están entremezcladas, pueden ser relativamente concebidas y enfocadas por separado. De entre las mismas, tres me parecen relevantes. La primera consiste en penetrar en los contenidos y preocupaciones que fueron emergiendo a través del tiempo, convirtiéndose en objetos principales de su búsqueda científica. Esta tarea conduce a explorar no solo los contenidos plasmados en su obra, sino en desentrañar la "lógica personal", el estilo de pensamiento y argumentación, coherencia metodológica y teórica; aspectos que a mi juicio han recibido relativamente poco tratamiento sistemático.

La segunda manera de aproximarse a este autor implica sumergirse en sus obras, en un intento por comprender significados, contenidos explícitos y subyacentes, esforzándose por descubrir las fases o etapas en que transcurrió su producción científica. Este es un trabajo sumamente importante que se ha extendido en el tiempo y que, hasta donde conozco, se está llevando a cabo de manera muy activa y productiva por un conjunto de estudiosos de la propuesta histórico cultural y particularmente del pensamiento de Vigotsky (González Rey, 2011; Kozulin, 1994; Van der Veer \& Valsiner, 1991; Wertsch, 1985; Závershnieva, 2014; entre otros).

Esta aproximación al legado de Vigotsky desemboca en la construcción y reconstrucción del aparato conceptual elaborado tanto por él como por parte de sus seguidores más directos, y presenta gran valor conceptual, teórico y metodológico al sentar las bases para la continuación del pensamiento histórico cultural, así como de otras orientaciones y perspectivas.

La tercera forma que tengo en mente, consiste en retomar las construcciones vigotskianas, tratar de extraer nuevos significados o significados no enfatizados por Vigotsky y hacer un esfuerzo por construir desde o sobre ellas. Este trabajo lleva a la formulación de nociones y construcciones en general deudoras (o así declaradas) del genio y la elaboración vigotskiana, pero que gozan de relativa independencia.

Desde luego, tratar de complementar y ampliar desde la obra de Vigotsky resulta una tarea sumamente difícil, sobre todo porque se debe hacer al alero de la genialidad y brillantez de su pensamiento. También, porque se corre el riesgo de no captar el verdadero significado de las categorías y construcciones que él avizoró; implicando el riesgo de no ser fieles a su pensamiento. Pero como ocurre en toda aproximación al pensamiento de predecesores geniales, la fidelidad a todo trance, por una u otra razón, puede conducir al inmovilismo que desemboca en la muerte de las ideas. En el caso de Vigotsky, sería una traición al eterno buscador y un acto ajeno a su profunda vocación dialéctica.

En concordancia con lo recién expresado, en este material quiero aproximarme a una de las nociones, a mi juicio, más importantes en la producción de Vigotsky: la zona de desarrollo proximal (ZDP).

De inicio trataré de enmarcarla dentro del pensamiento del autor, para después exponer algunos criterios acerca de lo que ha sido mi preocupación en los últimos tiempos: la noción de desarrollo en la ZDP y lo que debe hacer un estudiante implicado de manera verdaderamente activa (consciente y reflexiva) en una situación que propende a su desarrollo. Pienso que una de las dificultades más notables con que se ha enfrentado buena parte de los continuadores del legado de Vigotsky al trabajar la ZDP, es que lo han hecho desde la formulación directa realizada por él, con la intención de captarla stricto sensu, extraer implicaciones e instrumentalizarla en respuesta a la necesidades y demandas de la práctica formativa.

Gran parte de quienes han abordado la construcción de la noción de ZDP, se han detenido allí y no han tratado de analizarla a tono con las preocupaciones de Vigotsky que resultan centrales para comprenderla e incluso plasmarla en la enseñanza; por ejemplo, las formulaciones de la considerada como una de las etapas de su evolución científica (la tercera) donde sus intereses giraron en torno a la conciencia, el afecto, la vivencia y otras nociones por el estilo. De manera tal, que mientras el pensamiento de Vigotsky avanzaba hacia nociones como las antes citadas, que intentaban penetrar en la subjetividad, ofreciendo una aproximación más compleja, completa y real a la psiquis en desarrollo (González Rey, 2011, 2014), buena parte de sus continuadores no avanzaron correspondientemente en la exploración de concepto de ZDP desde esta dirección.

\section{La zona de desarrollo próximo según Vigotsky}

El interés de Vigotsky en la ZDP y su correspondiente abordaje, aparentemente ocurre en los años 1933-1934, en el curso de su motivación principal: aproximarse, de la manera más real posible, a la creación de una teoría de la interacción entre naturaleza y cultura, herencia y medio ambiente (Yasnitski, 2015).

Los años antes mencionados, marcan un momento donde predomina su preocupación por la relación entre aprendizaje y desarrollo; por conectar median- 
te una construcción teórica la cultura y los procesos de aprendizaje conducentes al desarrollo. Los autores han hecho un estudio bastante minucioso de esta etapa y la mayor parte concuerda en que luego de un período en que predominaron las aproximaciones de corte instrumentalista (González Rey, 2011; Zavershnieva, 2014), Vigotsky re(toma) sus preocupaciones en torno a la subjetividad, las emociones, la conciencia, el sentido, el significado, etc., generando construcciones que salen o van más allá de la problemática del desarrollo intelectual para penetrar hasta la personalidad como una categoría compleja y las connotaciones afectivas, valorativas y de sentido que le son concomitantes.

La noción de ZDP, como es conocida, se formula de manera explícita en el contexto del nudo de preocupaciones antes mencionado, aunque el propio Vigotsky no alcanza a insertarla de manera sustancial en el contexto de la subjetividad ni a trabajarla a fondo en el sentido de su instrumentación en la enseñanza. La concepción de ZDP, aparece abordada en pocas publicaciones (Vigotsky, 1993, 1996). En esas obras solamente está pespunteada la categoría y únicamente se bosquejan algunas consecuencias para la acción que refiere al desarrollo psíquico, que era la problemática que él tenía en mente. Sin embargo, la naturaleza de la interacción subyacente permite extraer un cúmulo de hechos de cardinal trascendencia.

La interacción en la ZDP, desde la preocupación de Vigotsky, está orientada al conocimiento del mundo y a la manera en que este se hace posible en el contexto de la interacción humana, en procesos específicamente organizados para ese objetivo (enseñanza) o en las situaciones cotidianas; los cuales tienen que ver no únicamente con la adquisición del conocimiento, sino sobre todo con el desarrollo del sujeto en ese proceso; pero, singularmente, penetra en el papel o la influencia del otro (más experimentado) durante el proceso de constitución del conocimiento y el advenimiento de su desarrollo. El papel del más experto consiste en aportar (organizar de cierta manera) los elementos que constituyen la ayuda o guía para promover el conocimiento del mundo y el desarrollo, habida cuenta de que ambos acontecen en el curso del enfrentamiento y la solución del problema.

Según Newman, Griffin y Cole (1991), al referirse a ZDP:

... el concepto se refiere a un sistema interactivo en el que varias personas se ocupan de problemas que, al menos una de ellas no podrá resolver sola. El cambio cognitivo se produce en esta zona, considerado tanto en términos de la historia evolutiva individual, como en los de la estructura de apoyo creada por los demás y por las herramientas culturales propias de la situación. (p. 77)

Los mismos autores también afirman que durante la acción en la zona, las interacciones estarán dominadas por las comprensiones de la persona más experimentada, que puede ser el maestro. Precisamen- te, esta parcialidad o asimetría en el conocimiento y conciencia de lo que ocurre en la zona, implícitamente señalada por los recién citados autores, es uno de los ángulos que trataré de abordar.

Volviendo a Vigotsky, aunque muchos autores destacan el matiz teórico de la ZDP no parece menos cierto que tras su formulación subyace una preocupación por proveer un asidero práctico a su construcción teórica sobre la relación entre aprendizaje y desarrollo. Precisamente, el exceso de énfasis que recibió la ZDP, particularmente, en cuanto a la manera en que podría ser instrumentada, es uno de los aspectos que posiblemente contribuyó a que fuera interpretada de una forma bastante distante de la preocupación sobre la conciencia y el significado, y que prevaleciera una visión sumamente instrumentalista de la misma.

Es patente que buena parte del interés de Vigotsky estaba orientado a proveer un anclaje metodológico (práctico) a las ideas sobre el desarrollo que él mismo había comenzado a elaborar. Este punto de vista lo expresa indirectamente Zavershnieva (2014) cuando apunta que "Además, en mi opinión, la psicología vigotskiana está en extrema necesidad de las teorías de nivel medio que podrían mediar entre, por un lado, la teoría y la metodología general y, por el otro, la práctica" (p. 31). Este planteamiento es extremadamente importante, sobre todo en lo que refiere a encontrar el "significado práctico" de la construcción, es decir, aquellas acciones, instrumentos y condiciones que pueden materializar, de la mejor manera, los aspectos que se plasman en la teoría. La ZDP es una de las nociones que abre la posibilidad de diseñar e investigar metodologías formativas para el desarrollo de los estudiantes en el proceso formativo de enseñanza y aprendizaje. Acaso mi intento se sitúa precisamente en la dirección que indica la autora.

Tanto el significado práctico aludido, como la posible apertura de la categoría de ZDP, pudieran buscarse en una formulación anterior de Vigotsky (1987), periodo sumamente polémico y considerado como predominantemente instrumental. Se trata de la idea de que, en el curso del desarrollo, la persona va adquiriendo el dominio de su propio comportamiento en el contexto de procesos mediacionales. Para él, el control se adquiere en el curso de un proceso que involucra de manera decisiva la acción guía que ejerce un sujeto más experimentado. Este, en el contexto de la enseñanza formal, se traza objetivos específicos y pone en práctica determinadas estrategias (pedagógicas) en el contexto de la enseñanza formal que supuestamente se hallan en correspondencia con la naturaleza de la actividad y le permiten alcanzar dichos fines. El dominio de la conducta es uno de los productos principales del desarrollo considerados por Vigotsky. Desafortunadamente, no lo elaboró en profundidad. 
Ligado a lo anterior, puede hacerse la pregunta de qué podría derivarse, a partir de la formulación que Vigotsky hace de la ZDP, como dominio de la conducta y cómo se ligan la comprensión y la conciencia a dicho dominio, sobre todo en contextos de desarrollo formalmente organizados. Al respecto, podría partirse de analizar la archiconocida definición, para destacar algunos aspectos que, más adelante, concitarán de manera detenida mi atención. Según Vigotsky, la zona:

...No es otra cosa que la distancia entre el nivel real de
desarrollo, determinado por la capacidad de resolver in-
dependientemente un problema, y el nivel de desarrollo
potencial, determinado a través de la resolución de un
problema bajo la guía de un adulto o en colaboración de
otro compañero más capaz. $(1988, p .133)$

Posteriormente, al comentar que la zona define más los "capullos" o "flores" que los "frutos" del desarrollo, pregunta "Entonces, ¿qué es lo que define la zona de desarrollo próximo, determinada por los problemas que los niños no pueden resolver por sí solos, sino únicamente con la ayuda de alguien? (Ibíd. p. 133).

Dos cosas destaco. Primero, que la noción de desarrollo que sirve de trasfondo, se refiere al desarrollo intelectual, concebido como la capacidad de abordaje y solución de situaciones y problema (en un principio, de forma asistida y luego de manera independiente). La segunda consiste en que el dominio podría referirse precisamente a la capacidad no ya de resolver el problema, sino de encontrar independientemente los medios de solución. Para ello es requerido orientarse y entender la situación, el problema; y desentrañar la relación en que respecto a él se hallan los medios y condiciones de solución; aspectos que antes fueron posible únicamente merced a la intervención del otro y que luego, al ser accesibles de manera independiente, dan fe del movimiento hacia adelante en el sentido del desarrollo (cognitivo).

Pero queda un asunto ¿el desarrollo entendido en términos de control de la conducta, o sea, del dominio de la situación, debería expresarse solo en función de la comprensión del problema y el alcance de la solución, aunque sea en su forma prospectiva, es decir como posibilidad de aplicación a situaciones de la misma familia, o podría entenderse también como comprensión de la situación en un sentido más holístico, en tanto que "situación de desarrollo", sus componentes y resultados, así como en la posibilidad de participar en su construcción?

En correspondencia con lo expresado, resulta viable la idea de que los cambios cualitativos que se producen en la conciencia y las interacciones de quienes participan en una situación que persigue el desarrollo de los sujetos pueden proveer criterios para estimar que el desarrollo ha tenido lugar.

Obviamente, entender el desarrollo de esta manera agregaría un peldaño a la comprensión y conocimiento de la situación, es decir, al control del comportamiento por el propio sujeto, al traer a primer plano la conciencia y la participación efectiva en la gestación y gestión de las condiciones que promueven el desarrollo (propio y de los demás).

\section{La interacción en ZDP}

Hace tiempo, autores como Rogoff (1990) expusieron la necesidad de que la interacción en la ZDP sea vista en diferentes planos que realmente actúan como una unidad. Uno de los aspectos resaltados radica en que en ella la adquisición de la competencia, la capacidad de actuar solo no es lo único importante, sino la construcción de sentidos que allí tienen lugar. La consideración del sentido como eje de la acción en la ZDP se halla en línea con la preocupación vigotskiana. En esta dirección, los autores han destacado consistentemente la zona como formación de la subjetividad, la orientación, la construcción de la conciencia y la emergencia de sentidos (Wertsch, 1985; Rogoff, 1990).

Tomando en consideración lo anterior y uniéndolo a la preocupación de Vigotsky en torno a la conciencia, el significado y el sentido factores esenciales en la construcción de la actuación y el desempeño del sujeto en situaciones que apuntan (explícita o implícitamente al desarrollo) puede decirse que comúnmente, la acción en la ZDP está diseñada más en concordancia con la solución de problemas en tanto que intencionalidad, que con el aumento progresivo del control del comportamiento vía participación en la construcción de la situación de desarrollo que representa.

En situaciones que impliquen desarrollo, mi idea es que el trabajo en la ZDP debe estar presidido precisamente por una doble orientación: la capacidad para resolver problemas de manera independiente $y$, a la vez, la posibilidad de participar en situaciones que impliquen desarrollo.

En el mismo sentido, considero importante el hecho de que la posición y la participación de los sujetos en los contextos de desarrollo, tiene que ver con la orientación u orientaciones predominantes, los significados que (se) construyan y la conciencia que alcancen de lo que realmente ocurre y su lugar en esas situaciones. Todo ello mediado por la acción de las personas más experimentadas, quienes realizan la función de guiar la actividad $y$, desde mi punto de vista, crean condiciones para que lo que ocurre en dichas situaciones se haga inteligible para los sujetos de menos desarrollo (Labarrere, 2000).

Por lo anterior, buena parte de la problemática ligada al desarrollo en la ZDP consiste en determinar la naturaleza de la acción guiada, cómo se construyen y elaboran dichos significados, la emergencia de la conciencia de los participantes menos experimentados y el 
tránsito a la participación independiente expresada en acciones colaborativas en los contextos de desarrollo.

\section{El desarrollo como capacidad de participar colaborativamente en las situaciones orientadas al desarrollo}

Una perspectiva para estimar el desarrollo de los sujetos en contextos y situaciones específicas orientadas a promover su desarrollo podría estar dada por la capacidad para incorporarse y participar de manera activa y consciente en la situación. Asumo que el surgimiento de la posibilidad de interactuar de manera colaborativa y consciente en dicha situación puede ser un indicador del desarrollo de los sujetos. ¿Es ello posible?

Quisiera recordar el hecho de que al formular la noción de ZDP, Vigotsky lo hace en términos de la ayuda y la colaboración, cuando dice que: "... bajo la guía de un adulto o en colaboración de otro compañero más capaz" (1988, p.133).

Al respecto, mi planteamiento central se remite a que, en la anterior formulación y otras relativas a la zona de desarrollo próximo que pueden encontrarse a lo largo de su obra, ayuda y colaboración están indiferenciadas, otorgándoseles la misma peculiaridad funcional respecto al aprendizaje y el desarrollo. Esto pudo deberse a que Vigotsky estaba interesado, sobre todo, en mostrar la necesaria participación del otro como representante de la cultura y la sociedad en el aprendizaje. En este sentido, es incuestionable que la ayuda y la colaboración son las formas fundamentales de actividad conjunta mediante las cuales los hombres interactúan. No obstante, en ocasiones, el autor reserva el término "colaboración" para la interacción de pares (los niños o alumnos entre sí). No parece haber considerado esta modalidad como cualitativamente diferente por sus efectos y susceptible de alcanzar una connotación muy específica al abordar la interacción que implica colaboración o cooperación.

Aunque no podemos tener total seguridad, es posible que, al no diferenciar ayuda de colaboración, y aplicar esta última, sobre todo, al caso de la interacción entre niños (alumnos), Vigotsky estuviera pensando que ellos, al menos formalmente, ocupan una "misma posición" desde la cual llevan a cabo su interacción. En el caso de la interacción alumno-adulto no sería así, pues este último interactúa con el primero desde la posición "más elevada" que le confiere su función social (deber de enseñar, transmitir experiencias, etc.). De ser cierta nuestra suposición, ello constituiría un elemento que favorece la distinción entre ayuda y colaboración al tratar el fenómeno del desarrollo.

Mientras la ayuda indica dependencia y asimetría, la colaboración define aspectos como propósitos compartidos, tal como John-Steiner, Minnis y Weber (1994, como se citó en John-Steiner, Weber \& Minnis 1998) propusieron:

The principles in a true collaboration represent complementary domains of expertise. As collaborators, not only do they plan, decide, and act jointly; they also think together, combining independent conceptual schemes to create original frameworks. Also, in a true collaboration, there is a commitment to shared resources, power, and talent: no individual's point of view dominates, authority for decisions and actions resides in the group, and work products reflect a blending of all participants' contributions... (p. 776)

Podemos cuestionarnos si al indiferenciar ayuda y colaboración, se eliminan aspectos con innegables repercusiones teóricas y metodológicas para la comprensión de lo que ocurre en la zona, así como para identificar peculiaridades de la interacción susceptibles de dar cuenta de formas y momentos relevantes del desarrollo de los sujetos en los contextos educacionales. Me inclino por una respuesta afirmativa, cuya aceptación puede dar inicio a una nueva manera de considerar lo que ocurre o debería ocurrir en la ZDP.

En aquellas aproximaciones que incorporan la ZDP de manera más estrechamente relacionada con sus posibilidades para la instrucción y la educación (como es la de este material), la ayuda y la colaboración no deben ser consideradas equipotentes por su función ni repercusiones en el desarrollo. Adicionalmente, sostengo que, en la interacción desarrolladora bajo los efectos de la ayuda, esta debe irse transformando paulatinamente en colaboración. Esto equivale a decir que, en la actividad educativa específicamente organizada y dirigida al desarrollo de los sujetos, una de las finalidades debe ser que las situaciones de ayuda en la zona, tiendan a devenir situaciones colaborativas para el desarrollo.

En línea con lo anterior, coincido con criterios como los de Steiner y Mahn cuando afirman que " the outcomes of... collaboration must be evaluated in context and over time" (p. 8). Tomando como base esta idea, podría derivarse que al estimar el desarrollo de los sujetos deben tomarse en cuenta no sólo criterios e indicadores relativos a su posibilidad de enfrentar, solucionar problemas y penetrar en las cualidades más internas de los objetos y fenómenos, sino también aquellos que hacen referencia a la naturaleza de la situación y a la participación en los procesos sociales interactivos que median la acción y están encaminados a promover el desarrollo de (todos) los sujetos implicados (incluso de aquellos considerados como de más experiencia). En ese sentido, considero que uno de los resultados de la interacción en la ZDP es precisamente el tránsito hacia formas interactivas más simétricas: de la ayuda a la colaboración.

Volviendo a la manera en que Vigotsky caracteriza la acción en la ZDP y sus resultados, prestemos atención a que refiriéndose al desarrollo él dice que lo que el su- 
jeto hoy hace con ayuda, mañana lo podrá hacer solo. Obviamente, se infiere que lo que mañana deberá hacer solo, sin ayuda, es resolver el o los problemas. Sin embargo, en concordancia con lo hasta aquí referido, es factible concebir que aparte la solución independiente de los problemas postulada por Vigotsky, el sujeto debería ser capaz de participar colaborativamente en pro del desarrollo; contribuyendo, incluso, a la creación de aquellas condiciones susceptibles de producir desarrollo, participando activamente en el diseño de situaciones virtualmente desarrolladoras. Desde luego, esta formulación implica aceptar de manera explícita que los sujetos participantes de procesos orientados al desarrollo pueden resultar capaces de operar en la situación con conocimiento y conciencia de la situación en que se hallan.

Analicemos primero la cuestión desde el punto de vista de su instrumentación y después desde la edad o momento de desarrollo.

La lógica del desarrollo debería concebir que gradualmente el estudiante adquiriese la posibilidad de orientarse y participar de manera consciente en la situación. Para ello se requiere cierta comprensión de la naturaleza de la situación, los objetivos para los cuales se ha diseñado e incluso la naturaleza de los mediadores que se han puesto en juego por parte de la persona más experimentada (Labarrere, 2000, 2015)

\section{Las situaciones de enseñanza y aprendizajes orientadas al desarrollo}

Por situación de enseñanza y aprendizaje orientada al desarrollo (SEAOD) debe comprenderse aquella donde el desarrollo de los sujetos intervinientes constituye el propósito principal (Labarrere, 2015). En tales situaciones, el desarrollo se estima en función de la posibilidad creciente de participar en procesos orientados a la transformación de los sujetos involucrados y de los contextos donde tiene lugar el desarrollo. En las SEAOD, el desarrollo es estimado a partir de la emer- gencia y transformación progresiva de la conciencia y el control (la producción) de la situación como una totalidad por parte de los sujetos, y la disposición para participar en la mencionada situación de una manera cada vez más efectiva.

Resulta evidente que las SEAOD son espacios interactivos, especialmente concebidos con la finalidad de producir transformaciones en los sujetos. Las situaciones enunciadas incorporan, al menos, los siguientes componentes funcionales:

a. Una o más finalidades u objetivos expresados en términos de desarrollo de los sujetos intervinientes.

b. Un conjunto de tareas y problemas que deben enfrentarse y resolverse.

c. Acciones que tienen por objeto el contenido y la solución de los problemas.

d. Un conjunto de estrategias, procedimientos y acciones de orientación e incorporación de los sujetos, las cuales promuevan progresivamente el tránsito de la ayuda a la colaboración.

Mientras las finalidades aportan el leitmotiv del desarrollo y confieren significado a la situación, las tareas o problemas constituyen el contexto para el desarroIlo, ofrecen la trama necesaria para que este pueda ocurrir. Las acciones que exploran el contenido de los problemas (sus condiciones y exigencias) tienen por finalidad desplazar el (los) sujeto(s) por el campo del problema o la tarea, garantizar la exploración del mismo, establecer las relaciones que lo constituyen y promover su solución efectiva. El último componente mira hacia la participación de manera específica, realiza la función de catalizador del tránsito de la ayuda a la colaboración, acompañando y permitiendo, en la medida de lo posible, que los sujetos menos expertos vayan ampliando progresivamente su conocimiento e incidencia en la situación de desarrollo (Ver Esquema 1).

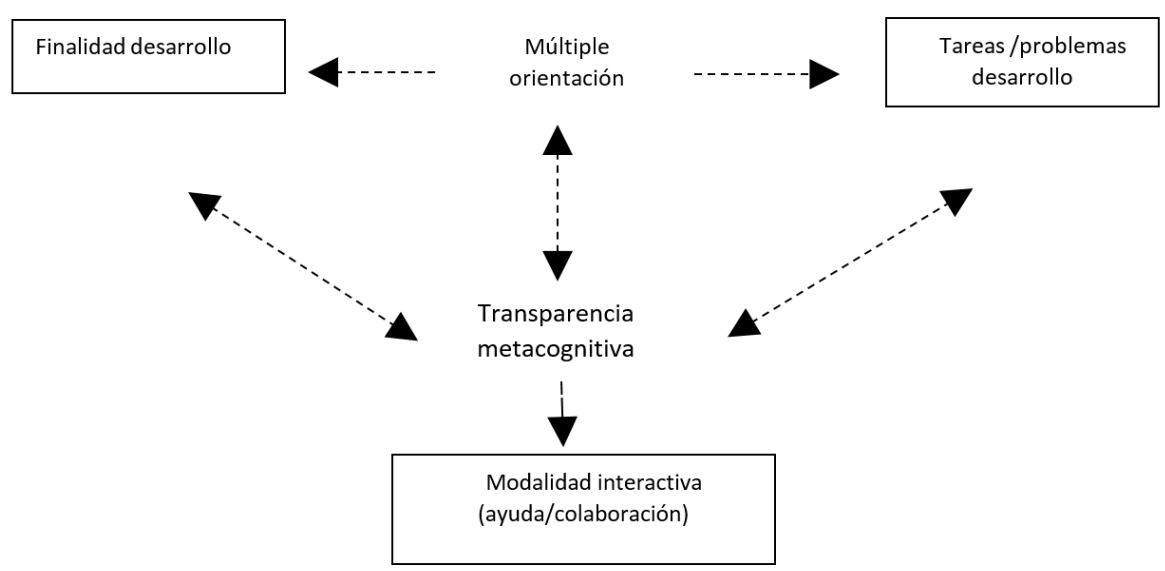

Esquema 1. Componentes estructurales de la acción en una SEAOD 
En una SEAOD, buena parte de las posibilidades del desarrollo de los sujetos menos expertos tiene lugar gracias a la asimetría que existe entre ellos y quienes en determinado momento asumen la responsabilidad de diseñar e implementar los procesos formativos. Por asimetría puede entenderse el desnivel o desfase relativo en los conocimientos, los recursos estratégicos y las representaciones de finalidades que aparecen cuando los sujetos interactúan en función del desarroIlo. Obviamente, si se expresara en términos de posibilidades para conducir los procesos, la asimetría pone a aquellos sujetos más experimentados en las mejores condiciones de diseñar, implementar, evaluar $y$, en suma, otorgar fundamento a la interacción dirigida al desarrollo. El monto o la carga de recursos que cada uno introduce en la situación se inclina decisivamente hacia el más experimentado, el guía, y se expresa mediante la identificación y formulación de problemas; no sólo los que refieren al contenido disciplinar involucrado, sino también aquellos que resultan de la misma acción formativa, tales como el diseño y dosificación de las ayudas introducidas, etc.

Dado lo anterior, es factible concebir que, durante el desarrollo de los sujetos en las SEAOD, se equilibran de manera gradual los aportes al desarrollo. Dicho de otro modo (empleando una metáfora), el monto de recursos que los sujetos colocan en la situación va tendiendo a hacerse más simétrico. En este caso, la simetría no significa estado de equilibrio, sino posibilidad de ofrecer aportes a la situación de desarrollo. El criterio de desarrollo no sería únicamente estimado en función de la solución de los problemas, sino por la forma en que el sujeto participa en la situación de manera global y la medida en que esta se va aproximando gradualmente a una genuina situación donde los intervinientes comparten recursos, colaboran.

Obviamente, la participación colaborativa en la SEAOD no resulta un mero hecho instrumental; conlleva, entre otros elementos, la conciencia de lo ocurre en dicha situación, el conocimiento y la proyección de finalidades, el significado de las acciones y del propio proceso que se vive, y la implicación en la situación. Esto genera la unidad de los factores cognitivos y afectivos concurrentes (González Rey, 2012; Mitjans, 2012; Andrade, 2012).

\section{La doble orientación en la ZDP como factor de emergencia de la colaboración en las SEAOD}

A partir de la argumentación que se ha realizado, en las SEAOD, el mañana de la colaboración y el aprendizaje para el desarrollo está signado por el manejo de instrumentos asociados explícitamente al desarrollo. Decir "explícitamente" implica que la acción en la ZDP debe propiciar ante todo la formación de la autoayuda, el autoandamiaje, y la autoasistencia para lo cual es necesario que los procesos formativos, por decirlo de una manera controvertida, sean muy densos en actos y orientaciones metacognitivas de los sujetos (Labarrere, 2000).

En correspondencia con lo anterior, la orientación al desarrollo puede concebirse como el conocimiento y la disposición para realizar el aprendizaje de los instrumentos diseñados para producir desarrollo; a saber, métodos, procedimientos, estrategias, etc., que promueven el desarrollo de las personas, los grupos, los contextos, etc. y que a su vez fomentan la puesta en acción. Este tipo de aprendizajes también ha sido denominado como aprendizaje de la mediación (Labarrere, 2008). Es aquí precisamente, donde lo que denominamos doble orientación u orientación multidimensional desempeña una función importante (Labarrere, 2003a).

Como se ha dicho, todo muestra que clásicamente, durante la acción en la ZDP la atención de los sujetos está fundamentalmente puesta en el problema que se resuelve y los medios de su solución. La doble orientación implica que el sujeto (el aprendiz en este caso) no concentra o dirige su atención únicamente al problema que se resuelve, tarea que se ejecuta, etc., alude a que no únicamente distribuye sus recursos en el análisis de la tarea, los objetos, las estrategias de solución, y así por el estilo, sino que también convierte en objeto de análisis, estudio y conocimiento la actividad de enseñanza; es decir, las acciones de los sujetos más experimentados (los profesores, la enseñanza, según sea el caso). Esa orientación hacia las acciones de los más expertos no suele ocurrir de manera espontánea (en la inmensa mayoría de los casos), tiene que ser trabajada explícita y directamente por la persona más experta.

De hecho, en las situaciones de enseñanza aprendizaje cotidianas, e igualmente en aquellas que pretendidamente se desarrollan a tenor la acción en la ZDP, como ya se dijo, la orientación de los sujetos menos experimentados se centra en la solución de los problemas. En tales condiciones, la orientación es, permítase la expresión, unidireccional en cuanto a que "se vira" (prácticamente, de manera exclusiva) hacia el plano del aprendizaje y tienen lugar, casi por entero, solo respecto al plano instrumental, esto es, respecto al contenido de la materia, los medios de solución, etc. (Labarrere \& Quintanilla, 2002).

Incluso en contexto de formación profesional, como es el de la formación de profesores, resulta común que los estudiantes presten atención consciente casi exclusivamente al contenido disciplinar $y$, sin embargo, no exploren de manera consecuente el modelo que expone el profesor, las acciones de enseñanza, el contexto donde se están produciendo la enseñanza y el aprendizaje. Estas se convierten en objeto de exploración consciente sólo cuando surge algún inconveniente y 
los análisis son casi siempre fragmentarios y relativamente unidireccionales. Este es un aspecto que será tratado con mayor detalle más adelante.

La noción de doble orientación tuvo sus inicios al final de los años 90 (Labarrere, 1999), cuando se comenzó a elaborar la idea de que, en el proceso de enseñanza y aprendizaje, los estudiantes aprendían sobre el mundo (las materias escolares, las ciencias, los procesos, hechos, etc., que les ofrece un cuadro científico del mundo), pero prácticamente nada respecto a sí mismos como aprendices y, menos aún, acerca de la actividad de enseñanza. La acción del profesor, el modelo que ofrece, prácticamente nunca es un objeto de exploración sistemática por parte de los estudiantes.

La idea era argumentar la importancia de que el estudiante no solo prestara atención a lo relacionado con el aprendizaje, sino también que explorara las acciones de los profesores y sus compañeros, que las hiciera objeto de su orientación como vía para formarse una representación más completa de la situación en que estaba inserto y alcanzar mayor comprensión de lo que estaba ocurriendo en el proceso de enseñanza y aprendizaje (considerado como una unidad). La asunción era que la orientación hacia la enseñanza constituía un requisito para mejorar el aprendizaje, particularmente, a los efectos de realizar acciones de aprendizaje independiente, repasar la materia, trazar metas a alcanzar en los diversos momentos, autoayudarse en el aprendizaje y prestar ayuda a los compañeros, etc., también como una manera de ejercer influencia sobre la enseñanza. Esto es una derivada y condición a la vez, de los procesos de colaboración.

\section{La Transparencia metacognitiva y el Triángulo interactivo como mecanismos propiciadores del tránsito de la ayuda a la colaboración en las SEAOD}

Con lo expresado en mente, consideré pertinente trabajar con lo que alguna vez denominé transparencia metacognitiva (Labarrere \& Quintanilla, 2002). Esta noción parte de considerar que, durante la enseñanza (la formación), los sujetos más experimentados (los profesores sea el caso) prácticamente nunca hacen explícitas las intencionalidades que se trazan y, sobre todo, aquellas que van surgiendo a lo largo del proceso de enseñanza. Esto ocurre igualmente en muchas situaciones orientadas al desarrollo donde los sujetos menos experimentados están al margen de las intencionalidades emergentes.

Ciertamente, en los espacios formativos (el aula, por ejemplo), al comienzo de las sesiones está normado que se realice la orientación hacia los objetivos como una actividad que persigue poner al tanto a los estudiantes de lo que va a ocurrir y prepararlos para que puedan intervenir adecuadamente en el proceso. Esta acción orientadora suele tener, además de la función de anticipación o activación cognitiva, otra motivacional que busca alcanzar un nivel óptimo de implicación en la tarea por parte de los estudiantes. Hasta aquí todo bien, pero resulta que es común que, en lo adelante, la lógica, la racional, de las acciones del profesor se esfume para los estudiantes (a veces también para él) y continúe un proceso donde no son evidentes los porqués de las acciones.

A partir de la asunción de que la colaboración requiere intencionalidades compartidas, acciones que se complementan y, como dicen John-Steiner, Weber y Minnis (1998), pensar conjuntamente la situación en que se está, es necesario que el estudiante esté en condiciones de pensar acerca de las acciones del profesor, tratar de hacerlas inteligibles, elaborar significados al respecto, etc. No obstante, la realidad indica que, en las condiciones actuales, este actuar sobre las acciones y el profesor no resulta factible para el estudiante. Esto debido, al menos, a tres razones: en primer lugar, porque en la lógica actual de una relación marcadamente asimétrica e impositiva, la actividad del profesor no siempre se abre al estudiante (jcada uno en su posición, OK!); en segundo lugar, porque la mirada centrada en el aprendizaje excluye el recurso de apertura de las acciones docentes (jno resulta necesario, pues el aprendizaje y la enseñanza transcurren bien sin que ello ocurra!) y en tercer lugar, porque los alumnos son incapaces de realizarlo (irecurso a la incapacidad... aprendida!).

Quisiera decir que, aunque me estoy refiriendo a las situaciones de enseñanza aprendizaje corrientes, no es muy diferente lo que ocurre en la ZDP. Como señalé en otra ocasión (Labarrere, 2003b), es común que, cuando se organiza la interacción desde la noción de la ZDP específicamente, los sujetos menos experimentados no tengan referencia concreta de lo que está ocurriendo respecto a su desarrollo. De este modo, la intencionalidad de desarrollo se les escapa y, tal cual he argumentado antes, transcurre en el plano meramente operativo, por lo que se les está escamoteando el acceso a la intencionalidad de desarrollo, así como a los productos del desarrollo.

Respecto a la noción de transparencia metacognitiva, debo decir que comencé caracterizándola como el intento consciente que hace el sujeto más experimentado (el profesor, sea el caso) por mostrar lo que denominé como códigos ocultos de la enseñanza. Quería aludir, precisamente, a las motivaciones que determinaban la realización de una u otra acción en el proceso de enseñanza, su por qué y para qué, cómo y cuándo, etc., tomando la iniciativa no solo al poner en conocimiento de los estudiantes la racionalidad de su acción, sino, sobre todo, al abrir su actividad a la exploración de estos. De esta manera, se conjugaba la acción de 
apertura, con finalidades orientadas hacia la cognición, implicación y emocionalidad de los estudiantes.

Con el paso del tiempo, comprendí que la transparencia metacognitiva, en un contexto que enfatizaba la simetría, no podía considerarse exclusivamente en términos del intento consciente del profesor por revelar los códigos ocultos de su actividad. Considerando la interacción profesor-estudiante como un sistema en evolución, la transparencia metacognitiva debía progresar cualitativamente e irse manifestando igualmente como intento consciente, sistemático y progresivo que lleva a cabo el estudiante para hacer explícitos los códigos ocultos de la actividad del profesor.

Obviamente, con semejante enfoque del desarrollo de la interacción, resulta posible investigar el progreso de los estudiantes y los profesores tomando como eje la disposición y capacidad para ocuparse de los códigos ocultos de sus acciones. Esto podría ser un indicador de desarrollo en términos de participación en las SEOD, así como una de las premisas y a la vez indicador del desarrollo de la acción colaborativa.

Ahora bien, si se asume como legítimo el hecho de que, en una SEAOD no ocurre espontáneamente la orientación de los sujetos (estudiantes) hacia las acciones de los profesores, ¿de qué manera es posible promover que dicha orientación tenga lugar? ¿Cómo puede provocarse y mediarse la misma? ¿Cómo puede mediarse la tendencia a poner de manifiesto los códigos ocultos de las acciones de los docentes?

Es aquí donde interviene la noción de triángulo interactivo. La denominación de triángulo interactivo ( $\mathrm{TI}$ ) está tomada en correspondencia con el clásico triángulo pedagógico de Houssage (1988), a la cual se le han incorporado sustanciales modificaciones (Lombard, 2007; Ibáñez, 2007). A finales de los años 90, concebimos una modalidad de interacción que incluía al profesor (o profesores), los estudiantes de dicha clase y una o más personas que presenciaban la clase y que tenían conocimientos de la materia e igualmente de pedagogía, (Labarrere, 1999). La modalidad interactiva consistía en que, durante la clase, los asistentes podían interactuar con el profesor del curso mientras este impartía la clase. Se trata de una modalidad cercana a la enseñanza en equipo y la enseñanza en equipo colaborativa, dado que puede incorporar diferentes personas en la ejecución de una actividad de carácter docente. La diferencia reside en que las personas incorporadas no participan en la preparación de lo que va a ocurrir en dicha actividad, y sus intervenciones se gestan al calor del desarrollo de la misma. ${ }^{1}$

El triángulo interactivo, a diferencia de la enseñanza en equipo, no solo estaba interesado en el aprendizaje

\footnotetext{
${ }^{1}$ EI TI como estrategia didáctica, se implementó en el Proyecto ARGOS para el desarrollo de la Inteligencia, la Creatividad y el Talento, en Ciudad Escolar Libertad, La Habana, Cuba
}

de las materias, sino que, sobre todo, perseguía que los estudiantes "mirasen", se orientaran hacia la enseñanza. Este, como se dijo en partes anteriores, es un requisito de la transparencia metacognitiva y de apertura hacia la colaboración. En este sentido, resultaba importante la participación de los asistentes en las actividades. Como se ha mencionado anteriormente, estos participantes tenían la posibilidad de dialogar con el profesor en momentos del desarrollo, exponiendo argumentos que podían atañer a la metodología $\mathrm{u}$ otros aspectos que estimasen convenientes. La introducción del diálogo, quebraba la lógica frontal del profesor como expositor y detentor único del conocimiento, mostraba a los estudiantes (y al propio profesor del curso) la relatividad del conocimiento y de las metodologías de enseñanza; los introducía en un contexto de problematización y polémica, donde la acción docente se exploraba y abría al cuestionamiento. Estamos hablando de clases de educación básica y media fundamentalmente.

La metodología seguida comprendía dos fases. En la primera, los estudiantes eran relativamente pasivos respecto de los análisis concernientes a la metodología u otros tópicos del contenido; su papel se limitaba a presenciar los intercambios entre los presentes, pero progresivamente se les iba haciendo partícipes de los episodios de análisis, con preguntas sencillas relativas a si se comprendió tal o cual explicación y si podían decir algo al respecto, etc. Debo decir que no se esperaban respuestas "correctas" o "no correctas", sino simplemente introducirlos en, hacerlos mirar hacia la enseñanza a través de la reflexión que tenía lugar.

Con el paso del tiempo, se observó como en momentos donde se sesionaba bajo la modalidad del triángulo interactivo, ya comenzaban a aparecer estudiantes que no aguardaban a que se les preguntara, e iban tomando la iniciativa de ofrecer su opinión respecto a uno u otro asunto de la enseñanza, de manera que con el tiempo, el intercambio entre profesores y estudiantes rompía el tradicional esquema de acción sustentado en preguntas y respuestas orientadas al contenido y se abría hacia aclaraciones, opiniones, preguntas del profesor y de los estudiantes acerca de la enseñanza. Los estudiantes comenzaron a indagar en torno al por qué de tal o cual manera de actuar del profesor y a hacer propuestas al respecto que eran analizadas conjuntamente.

Más cercanamente en el tiempo, y al alero de la Escuela de Psicología de la Universidad Santo Tomás, específicamente en la el área de psicología educacional y dentro de las Cátedras de Desarrollo de la Creatividad e Intervención Educacional, comencé a trabajar para orientar a los estudiantes hacia la enseñanza, haciendo objeto de su análisis sistemático la metodología de trabajo. En línea con ese cometido, se generan sesiones, en varios momentos del curso, específicamente 
destinadas al análisis metodológico. En estas sesiones, los estudiantes realizan la revisión de la metodología de enseñanza, plantean lo aspectos deficitarios y los logros principales, expresan y discuten sugerencias relativas a cómo trabajar tal o cual tópico, etc.

No menos importantes resultan las acciones destinadas a la reflexión y el desarrollo de la conciencia de esa manera de actuar; acciones que van dirigidas y penetran en el plano personal significativo (Labarrere \& Quintanilla, 2002) al actuar sobre el sistema de significados que los estudiantes construyen, la implicación y el compromiso con las materias y la metodología. Todos estos constituyen elementos que, según Vigotsky, resultan cruciales en los hechos del desarrollo especifico con respecto a la conciencia como sistema de significados, los cuales se construyen y transforman en el curso de la actividad.

En las sesiones, los estudiantes analizan la metodología y adicionalmente, la relacionan con sus propios aprendizajes, dificultades, progresos, etc., haciendo propuestas al respecto. La sistematización de las sesiones constituye un factor crucial en el desarrollo de la orientación hacia la enseñanza y el aumento de su participación y responsabilidad en la misma; aspectos claves para el desarrollo de la colaboración, tanto en términos de acción instrumental como desde el ángulo de la responsabilidad e implicación de los estudiantes.

Otra estrategia que actualmente se implementa a nivel general, en el área de psicología educacional, son las sesiones de reflexión y análisis metodológico (SERAME). Estas sesiones están dedicadas al trabajo metodológico, al análisis de las metodologías empleadas por los docentes en las diversas situaciones de enseñanza. En ellas un profesor hace propuestas relativas a la manera de abordar un determinado tópico o contenido perteneciente al programa. El resto de los presentes tiene la posibilidad de realizar análisis de la propuesta, hacer sugerencias, destacar debilidades y fortalezas, plantear variantes, etc., pero la peculiaridad radica en que dichas sesiones son abiertas a los profesores que quieran participar (no sólo del área), sino también o sobre todo, a los estudiantes. Obviamente, las SERAME constituyen variantes del triángulo interactivo antes tratado.

Las SERAME son otra vía para poner a los estudiantes en condiciones de "mirar", orientarse hacia, hacer la exploración de la enseñanza y participar constructivamente en ella. Esto como forma de desarrollar la colaboración con los profesores y con los compañeros en ocasiones de trabajo conjunto, preparación colegiada de la materia, etc.

Tal como expresé hace mucho tiempo (Labarrere, 1999), la orientación hacia la enseñanza no resulta irrelevante al aprendizaje y el desarrollo, creo que es una condición indispensable para un desempeño consciente y verdaderamente participativo en situaciones formativas que, aparte de hacia el aprendizaje del contenido, se propongan el desarrollo de quienes en ellas están insertos (sujetos más y menos expertos) para generar verdaderos procesos colaborativos; para transitar de situaciones donde los menos experimentados (estudiantes) esperan y se anclan en la ayuda del profesor hacia otra donde aparece la independencia y la posibilidad de actuar conjunta y colaborativamente no sólo con los compañeros, sino también con los profesores.

La formación de la colaboración en el curso del aprendizaje y la enseñanza es de importancia superlativa para todos los estudiantes de nivel superior, pero sobre todo para aquellos que se forman en profesiones como el magisterio y la psicología. Al respecto se ha de señalar que quienes estudian para estas profesiones (incluso cuando tienen un bagaje relativamente amplio de conocimientos y competencias conectadas a la enseñanza y al funcionamiento humano), solo en contadas ocasiones ponen estos conocimientos en función del mejoramiento de lo que ocurre en los espacios formativos.

Mi experiencia indica que los estudiantes de los últimos niveles de la formación profesional en las dos carreras que he mencionado casi nunca realizan por propia iniciativa un análisis de lo que ocurre en la formación desde una perspectiva profesional, valga decir, aplicando las teorías que han estudiado. Por el contrario, más bien ejecutan este análisis de manera intuitiva (lo cual no corresponde con un profesional que se forma en la especialidad) y solo cuando se generan ante ellos barreras a la formación, las cuales se suele atribuir únicamente a la acción del docente.

Obviamente, la posibilidad de actuar sobre la enseñanza depende muchas veces de factores externos al estudiante y al propio profesor. Por ejemplo, currículos, programas y cronogramas, que no comparten o incorporan la posibilidad de que los estudiantes realicen aportes a lo planeado, constituyéndose en esquemas rígidos que, a la larga, no son consecuentes con la lógica de la función desarrolladora de la enseñanza. Aunque la aludida función suele ser asumida por los docentes, hay evidencias de investigaciones (Malvaez \& Labarrere, 2015) que muestran como lo expresado en el discurso muchas veces no es corroborado por la acción práctica.

\section{Una vuelta al lazo de la ZDP}

Tal vez estoy muy lejos de Vigotsky y la ZDP. Tal vez no. Pienso que estoy cerca porque, desde mi punto de vista, todo lo que he planteado se refiere al desarrollo, a la tentativa por mediar ese desarrollo como objetivo central de la enseñanza y de la formación en general, preocupaciones permanentes en aquel genio. La ZDP es 
una zona donde no solo se construye el conocimiento; en ella también se forma la colaboración y la conciencia, y con ambas, toda una serie de características de la persona(lidad) que Vigotsky tenía en mente desde sus primeras producciones y que retomó con énfasis y fortaleza renovados poco antes de su deceso.

Pienso que la noción de SEAOD resuena dentro de la lógica de la ZDP y se abre paso como un intento de construir a partir de esta última. Los ejemplos y situaciones reportados, los concibo a medio camino entre la acción en la ZDP (organizada bajo un relativo control) y las situaciones de carácter más abierto donde los procesos mediadores y las acciones correspondientes se resisten al control, la anticipación y se abren hacia la incertidumbre y la elaboración in situ o en contextos emergentes.

\section{Referencias}

Andrade, V. (2012). Subjetividade, aprendizagem e desenvolvimento de estudantes do Ensino Superior. En A. Mitjans (Ed.), Ensino e aprendizagem: a subjetividade em foco (pp. 183-201). Brasilia: Liber Livro.

González Rey, F. (2011). El pensamiento de Vigotsky. Contradicciones, Desdoblamientos y Desarrollo. México: Trillas.

González Rey, F. (2012). A configuração subjetiva dos processos psíquicos: avançando na compreensão da aprendizagem como produção subjetiva. En A. Mitjans (Eds.), Ensino e aprendizagem: a subjetividade em foco (pp. 21-41). Brasilia: Liber Livro.

González Rey, F. (2014). Advancing further the history of soviet Psychology: moving forward from dominant representations in western and soviet psychology. History of Psychology, 17(1), 60-78. doi:10.1037/a0035565

Houssage, J. (1988). Le triangle pédagogique. Berna: Peter Lang.

Ibáñez, C. (2007). Un análisis crítico del modelo del triángulo pedagógico. Una propuesta alternativa. Revista Mexicana de Investigación Educativa, 12(32), 435-456. Recuperado de http://www. redalyc.org/pdf/140/14003220.pdf

John-Steiner, V., Weber, R. \& Minnis, M. (1998). The Challenge of Studying Collaboration. American Educational Research Journal, 35(4), 773-783. Recuperado de http://www.unm.edu/ vygotsky/chall.pdf

Kozulin, A. (1994). La psicología de Vigotsky: Biografía de unas ideas. Madrid: Alianza.

Labarrere, A. \& Quintanilla, M. (2002). La solución de problemas científico en el aula. Reflexiones desde los planos de análisis y desarrollo. Pensamiento educativo, 30(1), 121-137. Recuperado de http://pensamientoeducativo.uc.cl/index. $\mathrm{php} / \mathrm{pel} / \mathrm{article} / \mathrm{view} / 216 / 453$

Labarrere, A. (1999). Aprendizaje...¿Qué le oculta la enseñanza?. Siglo XXI, 1(7), 36-44. Recuperado de http://www.docfoc.com/labarrere-aprendizaje-que-le-oculta-la-ensenanza

Labarrere, A. (2000). Aprendizaje para el desarrollo. Revista cubana de psicología, 17(1), 28-30. Recuperado de http://www7.uc.cl/sw educ/educacion/grecia/plano/html/pdfs/Formacion continua/talleres/FTA003.pdf

Labarrere, A. (2003a). Funcionamiento cognitivo y desarrollo en ZDP. Pensamiento educativo, 32(1), 141-154. Recuperado de http://pensamientoeducativo.uc.cl/index.php/pel/article/ view/250/520

Labarrere, A. (2003b). La formación de profesores de ciencia: un enfoque desde la teoría de la profesionalidad temprana y los procesos de orientación. Revista Extramuros, 2, 21-33.

Labarrere, A. (2008). Bases conceptuales de la mediación y su importancia actual en la práctica pedagógica. Summa Psicológica UST, 5(2), 87-96. doi:10.18774/summa-vol5.num2-214

Labarrere, A. (2015). Desarrollar la creatividad de los estudiantes de educación superior:desafío del siglo XXI. Santiago de Chile: Universidad Santo Tomás.

Lombard, F. (2007). Du triangle de Houssage au tétraèdre des TICS. En B. Charlier \& D. Peraya, Transformatios des regard sur la recherche en technologie de l'education (pp. 137-154). Louvain: de Boeck Superieur.

Malvaez, O. \& Labarrere, A. (2015). Concepciones del profesorado de ciencia en ejercicio acerca del desarrollo. Una aproximación desde la representación de su práctica. Santiago de Chile: Editorial Académica Española.

Mitjans, A. (2012). Aprendizagem criativa:uma aprendizagem diferente. En A. Mitjans (Ed.), Ensino e Aprendizagem: A subjetividade em foco (pp. 85-1109). Brasilia: Liber Livro.

Newman, D., Griffin, P. \& Cole, M. (1991). La zona de construcción del conocimiento: trabajando por un cambio cognitivo en la educación. Madrid: Morata S.A.

Rogoff, B. (1990). Apprenticeship in thinking: Cognitive development in social context. New York: Oxford University Press. 
Van der Veer, R. \& Valsiner, J. (1991). Understanding Vygotsky: A Quest for Synthesis. Oxford (UK), Cambridge (USA): Blackwell.

Vigotsky, L. (1996). El desarrollo de los procesos psicológicos superiores. Barcelona: Crítica.

Vigotsky, L. (1987). El desarrollo de las funciones psicológicas superiores. La Habana: Universitaria.

Vigotsky, L. (1993). Estudio del desarrollo de los conceptos científicos en la edad escolar. En L. Vigotsky \& A. Álvarez (Eds.), Obras escogidas Tomo II (pp. 9-348). Madrid: Visor.

Vygotsky, L. (1988). Thought and Language. Cambridge: The Massachusetts Institute of Technology.

Wertsch, J. (1985). Vigotsky and the social formation of mind. Cambridge: Harvard University Press.

Yasnitsky, A. \& Van der Veer, R. (2015). Revisionist Revolution in Vygotsky Studies: The State of the Art. New York: Routledge.

Závershnieva, Y. (20 de diciembre de 2014). El problema de la consciencia en la psicología histórico-cultural de Vigotsky. Recuperado de http:// vygotski-traducido.blogspot.cl/search/label// Consciencia 